\title{
A Portable 3D Body Scanner and its Application
}

\author{
Hideto KAMESHIMA ${ }^{a}$, Masaki HAYASHI ${ }^{a, b}$, Yuji NISHIO ${ }^{a}$, Yukio SATO ${ }^{a, b}$ \\ ${ }^{a}$ Spacevision Inc., Tokyo, Japan; \\ ${ }^{\mathrm{b}}$ Keio University, Kanagawa, Japan
}

\begin{abstract}
A portable, ultra-compact, and high-speed scanner for 3D human body scanning is described in this paper. The new "separable and easy to carry out" 3d body scanner is called," Traveling Type". The portability of it must cultivate new uses of $3 d$ body scanning technologies.
\end{abstract}

Keywords: 3d body scanning, portable, high-speed, smart calibration

\section{Introduction}

A portable, ultra-compact, and high-speed scanners for 3D human body scanning is described in this paper. The 3D Body Scanner Traveling Type, shown in figure.1, is an unprecedented "portable" 3D scanning system which can be disassembled into nine portable projector-camera components.

Spacevision is a leading company for portable 3D human body scanner, which is called "Cartesia 3D Body Scanner", which is a separable and portable system. In this paper, we present our new "separable and easy to carry out" $3 d$ body scanner, which is called," Traveling Type". The portability of it must cultivate new uses of $3 d$ body scanning technologies.

\section{System}

The system consists of three tower units (four tower units type is also available). Each tower unit has three vertically stacked projector-camera components and a pedestal (see figure.1). The dimensions of each projector-camera component are $7 \mathrm{~cm}$ width, $20 \mathrm{~cm}$ depth and $65 \mathrm{~cm}$ height. The total height of the assembled tower unit is about $200 \mathrm{~cm}$. The weight of each component is only $3.1 \mathrm{~kg}$, so that it can be carried separately with a suitcase by one person.

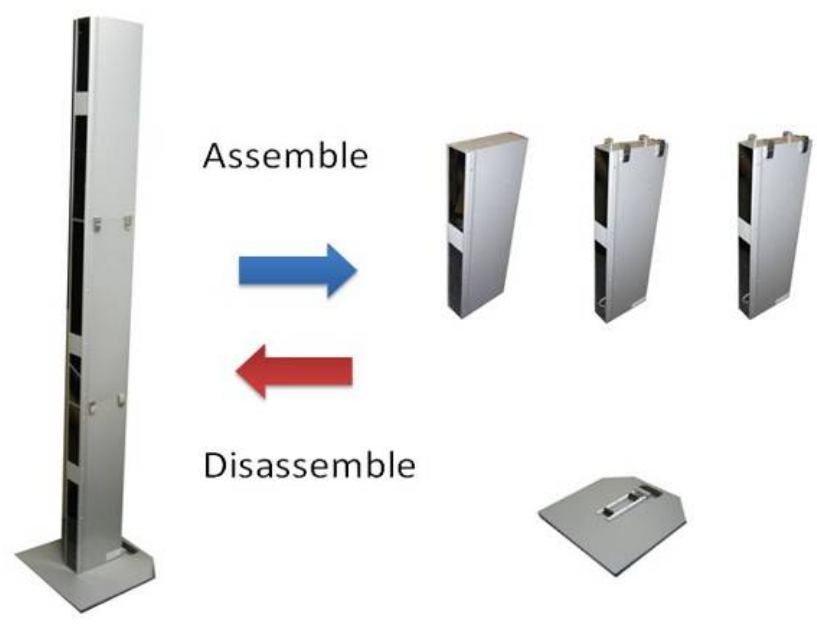

Fig.1. Components of Portable 3D Body Scanner. Left: The whole system. Right: Each tower can be disassembled into 3 pro-cam modules. You just need to stack up modules to (re-)assemble them. 


\subsection{Portability and Compactness}

Portability for $3 \mathrm{~d}$ body scanners had been highly demanded from several kinds of industries for long, but never achieved before. The system realizes a great portability and compactness with following two solutions:

(1) Decomposable into portable units.

As already mentioned before, each tower of the system can be disassembled into 3 modules, and each module is easy to carry. Therefore, users can easily move or transport the whole system to another place by disassembling it into 9 parts.

(2) Adjustable with multi-camera calibration whenever you replace your system.

Multi-camera calibration must be done by users after placing all tower units onto their site. Namely, each projector-camera modules has its own projector-camera parameters calculated before shipments, so all you have to do is just perform multi-camera calibration, which only takes about 5 minutes, after placing the system onto your site. This calibration procedure enables you to replace the system to another site as many as you like. We strongly believe that this portability can extend how and where to use $3 d$ body scanners.

\subsection{High Speed Capture with colored texture}

The system measures the 3D human body shape and its color (see figure 2) within 2.0 seconds owing to its fast capturing of a range image. This high speed capturing is derived from multi-camera calibration performed in advance. Measurement density can be increased up to 1 million points or more with keeping the average margin of error within $3 \mathrm{~mm}$ or less.
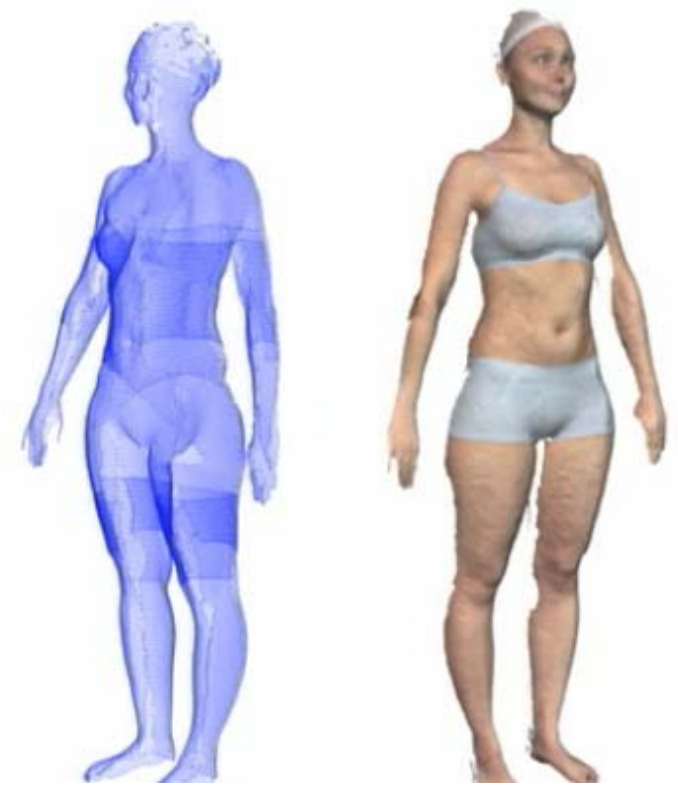

Fig.2. Measured Human Body. Left: Point cloud without texture. Right: Textured point cloud

\subsection{Easy setup}

Setup is also easy for even an unskilled operator. First, the bottom projector-camera component is placed on the pedestal and fastened by a wrench. Next, the middle component and upper component are laid on the bottom component in order. The viewpoints of the nine projector-cameras in each tower units are arranged beforehand in order to measure the human body with less occluding parts. Careful or precise placement of the tower units is not needed because calibration process assures the accuracy of the synthesis of the data taken from each tower units. Moreover, the calibration procedure of projector-camera parameters is quite simple and finishes quickly. 


\section{Applications}

The measured data obtained via Cartesia 3D body scanner can be incorporated into 3D body scanning software developed by $[T C]^{2}\left({ }^{*} 1\right)$. The $3 \mathrm{D}$ body scanning software of $[\mathrm{TC}]^{2}$ models a human body shape from the measured point-cloud data, and automatically carries out landmarking and measurement. The software also corresponds to color texture mapping. This software product has already been widely used in a variety of countries as one of the worldwide standard software.

In addition, i-Fashion ( $\left.{ }^{*} 2\right)$ in Korea has developed a software to utilize measured data obtained via Cartesia 3D body scanner. Furthermore, Hokuriku STR $\left({ }^{\star} 3\right)$ has developed software which integrate processes from 3D scanning to body shape analysis by utilizing our SDK (Software Development Kit) for body scanner control.

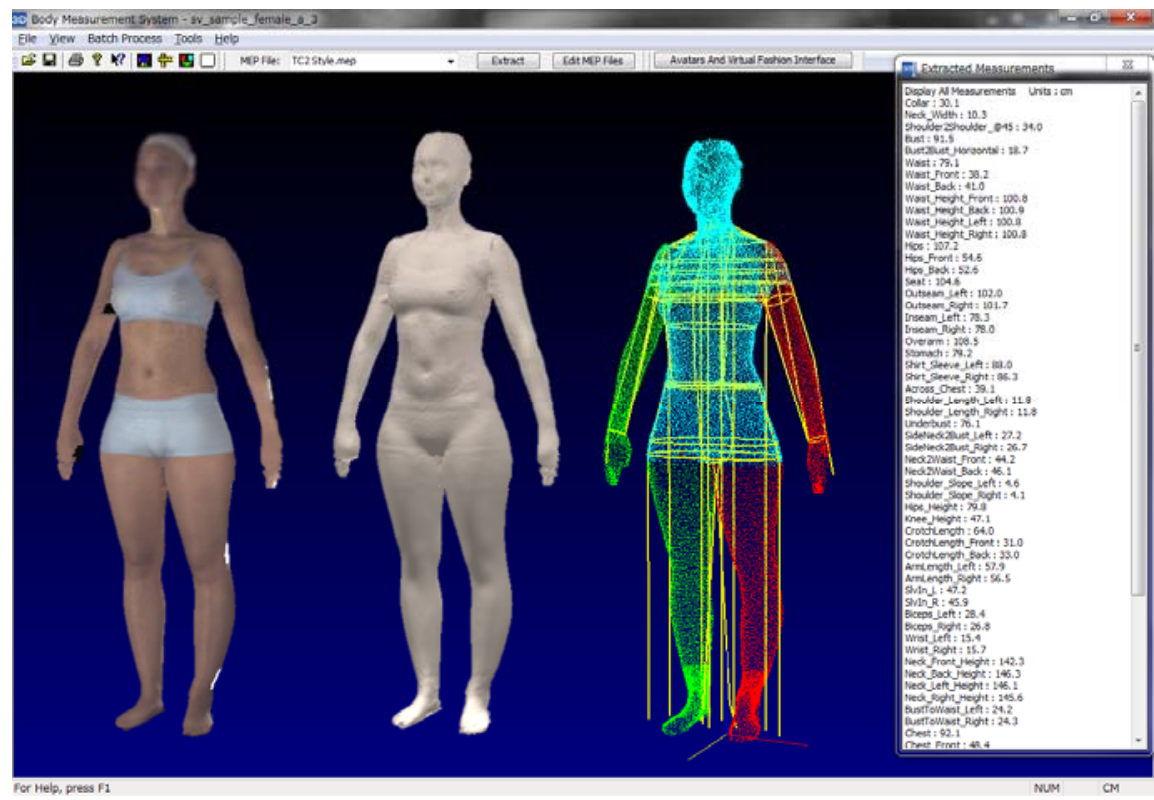

Fig.3. The measured data obtained via Cartesia 3D body scanner is incorporated into $3 D$ body scanning software of $[T C] 2$.

\section{References}

1. $[\mathrm{TC}]^{2}$ : http://www.tc2.com

2. i-Fashion: http://www.ifashion.or.kr/

3. Hokuriku STR Cooperative, "Cousette": http://www.str.or.jp/cousette 